\section{Organisationales Lernen und betrieblicher Umweltschutz}

Sieht man es als Aufgabe der Betriebswirtschaftslehre an, die in der Praxis entstehenden Probleme von Betriebswirtschaften theoretisch zu deuten und auf dieser Basis Gestaltungsvorschläge zur Optimierung von Betriebswirtschaften zu unterbreiten, dann ist ohne Zweifel der betriebliche Umweltschutz zu einem wichtigen Problemfeld avanciert. Entgegen frühen Annahmen, wonach eine ethisch-normative Betriebswirtschaftslehre - der Praxis vorausgehend - Entwürfe eines umweltverträglichen Wirtschaftens zu unterbreiten habe (vgl. hierzu kritisch Ridder 1986; 1990; 1993), erweisen sich heute Fragen des betrieblichen Umweltschutzes als schnell wachsende Herausforderung im Rahmen der praktisch-normativen Betriebswirtschaftslehre.

Die Ursache dieser zunehmenden Beschäftigung der Betriebswirtschaftslehre mit Fragen des betrieblichen Umweltschutzes dürfte im Erklärungs- und Beratungsbedarf der Praxis liegen, die Umweltschutz verstärkt als Risiko und gelegentlich als Chance zur Kenntnis nehmen muß.

\section{Spezifische Selektivität der Umwelt}

Geht man von der Annahme aus, daß Unternehmen den betrieblichen Umweltschutz in erster Linie dann als Herausforderung begreifen, wenn durch den Markt oder durch Anspruchsgruppen Umweltschutz betriebswirtschaftlich relevant wird, dann ist die Antizipation einer in dieser Hinsicht ungewissen Zukunft von den Unternehmen in gleicher Weise zu leisten, wie die Antizipation von Technologien oder Veränderung von Märkten. Dabei erschließt sich Umwelt den Unternehmen nicht von selbst, sondern tritt den Unternehmen ebenfalls in einer spezifischen Selektivität gegenüber:

Unternehmen interpretieren Umweltschäden als externe Herausforderung, die unter Umständen im Rahmen der Strategiekonzeption zu berücksichtigen ist. Dies geschieht höchst differenziert und häufig in Abhängigkeit von der ökonomischen Betroffenheit der Unternehmen. Betrieblicher Umweltschutz bedeutet damit zunächst die Identifikation der Herausforderung, die das Unternehmen zu bewältigen hat. In Szenarien hätten die Unternehmen zu prüfen, ob diese Herausforderung durch den Gesetzgeber, die Arbeitnehmer oder die Konsumenten verursacht wird, um die Richtung notwendiger Maßnahmen frühzeitig ordnen zu können. Bereits an dieser Stelle wären Entscheidungen zu treffen, ob z.B. das Personalentwicklungsbudget eher in die Entwicklung und Anwendung von Umweltschutztechnologien, Entwicklung und Beschaffung alternativer Roh-, Hilfs- und Betriebsstoffe (Bedrohung durch den Gesetzgeber) oder in Marktaktivitäten (Öko-Image, Verkäuferschulung etc.) zu investieren ist. Positiv formuliert könnte die Unternehmung durch die strategische Berücksichtigung des Umweltschutzes nicht nur die Abwendung von Bedrohungen durch externe Anspruchsgruppen antizipieren, sondern den langfristigen Aufbau strategischer Vorteile realisieren.

Konsequenterweise ist allerdings darauf hinzuweisen, da $\beta$ weder strategische Planung noch die Beschäftigung mit Fragen des Umweltschutzes in den Unternehmen sehr weit verbreitet sind. Offensichtlich ist es bislang nur in geringem Ausmaß gelungen, Unternehmen von der Vorteilhaftigkeit dieser Maßnahmen zu überzeugen.

Der Beurteilung des betrieblichen Umweltschutzes als strategierelevantes Thema folgt die Frage, wie die Unternehmung diesen heterogenen Bereich im Unternehmen etablieren soll, wie insbesondere die Lernfähigkeit der Mitarbeiter bzw. der gesamten Organisation im Hinblick auf den betrieblichen Umweltschutz verbessert werden kann.

Allgemein besteht das Ziel organisationalen Lernens - folgt man Dodgson (1993) - darin, in Zeiten großer Unsicherheit Anpassungsmöglichkeiten zu verbessern. Interne Faktoren sollen diese Herausforderungen erkennen und in einem wechselseitigen Stimulierungsprozeß synergetische Anpassungsprozesse erlauben. Aus diesem Grunde hat es bspw. in der Betriebswirtschaftslehre eine umfassende Diskussion über Strategieentwicklung, Veränderung von Strukturen und die Rolle des Menschen als flexiblen Anpassungsmechanismus gegeben. Hier geht es darum, durch strategische Unternehmensführung Ungewißheiten innerhalb von Szenarien zu antizipieren, Organisationsstrukturen diesen Herausforderungen anzupassen und die personalwirtschaftlichen Instrumente nicht mehr als gewohnheitsmäßigen Appendix zu begreifen, sondern vielmehr eine Verbindung herzustellen zwischen der antizipierten Zukunft und den in dieser Hinsicht frühzeitig zu bestimmenden personalwirtschaftlichen Ressourcen.

\section{Die neue Botschaft: organisationales Lernen}

Diese notwendigen Veränderungen aktiv zu betreiben, bedarf allerdings einer gewissen $\mathrm{Be}-$ reitschaft und Fähigkeit der Organisation und ihrer Mitglieder. Organisationsforscher suchen daher nach den Bedingungen der Möglichkeit, die notwendigen Veränderungsprozesse möglichst friktionslos in den Organisationen zu implementieren und durchzusetzen. Nach Personalentwicklung und Organisationsentwicklung heißt die neue Botschaft: organisationales Lernen. Diese neue Metapher kann als Antwort auf die turbulenter gewordene Dynamik gewertet werden. Sie signalisiert Engagement, Fortschritt, Bereitschaft zur Veränderung.
Ausgehend von den klassischen Konzepten des single-loop learning und double-loop learning (Argyris, Schön 1978) wurde bald eine Vielzahl von Konzepten des organisationalen Lernens entwickelt, die mehr oder weniger alle ihren Schwerpunkt auf die Unterscheidung zwischen drei Ebenen legen (vgl. ausführlich Pawlowsky 1992):

Auf der ersten Ebene konzentriert sich die Organisation auf die Identifikation und Behebung von Normabweichungen. Veränderungen oder negative Rückkoppelungen werden mit Hilfe der gültigen Organisationsmechanismen beantwortet. Modifikationen im. Lernsystem sind als Adaptionsprozeß möglich, ohne jedoch die Grundstruktur des Lernprozesses zu verändern.

$\checkmark$ Auf der zweiten Ebene unterzieht die Organisation ihre Handlungstheorien in Konfrontation mit Umweltbeobachtung einer kritischen Revision. Möglich sind Veränderungen der Weltbilder von Organisationsmitgliedern. Die Suche nach neuen Lösungsmöglichkeiten bestimmt das Problemlösungsverhalten. Dennoch bleibt der Lernprozeß reaktiv, da auch hier ein von außen wirkender Einfluß diese Suche maßgeblich beeinflußt.

- Auf der dritten Ebene wird die Modifikation der Lernfähigkeit einer Organisation zum Gegenstand von Lernprozessen. Zum Gegenstand wird auch die Reflexion der eigenen Handlungen. Erstrebt wird ein Verständnis für die Prinzipien und Zusammenhänge des organisationalen Lernens, des Aufbaus und der Pflege des organisationalen Wissensbestandes.

Wichtig für den folgenden Gedankengang scheint mir, daß organisationales Lernen keine unbestimmte Größe darstellt, sondern seinen Inhalt erst durch die Explikation von Zielen gewinnt. Organisationen hätten demnach zu prüfen, welche Ziele sie im Zusammenhang mit organisationalem Lernen verfolgen, wie der Pro$\mathrm{ze} ß$ des organisationalen Lernens organisiert werden kann und welche Faktoren das organisationale Lernen unterstützen.

Bezieht man diese Fragen auf die umweltpolitischen Herausforderungen, denen sich Unternehmen heute stellen, können drei Fälle unterschieden werden:

- Identifiziert die Unternehmung umweltpolitische Herausforderungen, kann sie mit Anpassung der Routinen reagieren. Im Hinblick auf akute und eindeutig spezifizierte Herausforderungen können Personen akquiriert oder entwickelt werden, in deren Arbeitsbereich spezifische umweltbezogene Herausforderungen eingetreten sind oder in naher Zukunft eintreten (z. B. Teilbereiche der Produktion, des Absatzes, aber auch PR-Maßnahmen etc.). Zu denken ist hier an die verstärkte Ausbildung von Technikern im Bereich des Umweltschutzes, die Not- 
wendigkeit der personellen Professionalisierung im Hinblick auf Gesetze, Verordnungen, Genehmigungsverfahren etc. Das Auswahlkriterium wäre hier die Identifikation der umweltschutzbezogenen Herausforderung der Unternehmung und die Auswahl sowie Budgetierung von $\mathrm{Maßnahmen}$ zur Personalentwicklung. Die Organisation verändert hier weder ihre Routinen noch die grundsätzliche Denkhaltung. Auf Herausforderungen, die durch den Umweltschutz generiert werden, wird in gleicher Weise reagiert wie auf technologische̊ oder durch den Markt induzierte Herausforderungen.

- Im Bereich des Umweltschutzes können aber nicht alle Herausforderungen eindeutig spezifiziert werden. In Abhängigkeit von der Gefährdungslage der Unternehmung lassen sich häufig zunächst Handlungsfelder nur grob identifizieren, die das jeweilige spezifisch ökonomische Interesse dokumentieren. Es gilt dann, frühzeitig Schlüsselpositionen oder Gruppen zu bestimmen, die bestehende Routinen überprüfen und im Sinne der strategisch vorgedachten Maßnahmen nach neuen Wegen der Bewältigung von Herausforderungen suchen und eine Verbreitung der geplanten Maßnahmen unterstützen. Es geht also um die Etablierung von neuen Problemlösungsmechanismen.

Als Beispiele sind hier Projektgruppe oder Lernstatt zu nennen, die eine Einbringung der Ideen der Arbeitnehmer bei der Suche nach neuen Wegen zur Bewältigung der umweltpolitischen Herausforderungen erlauben. In Praxisberichten wird immer wieder deutlich, $\mathrm{da} B$ das Engagement und die Phantasie der Arbeitnehmer teilweise zu erheblichen Verbesserungen der Kostenstruktur der Unternehmen geführt haben. Auch die zunehmende Verbreitung des
Umwelt-Audit setzt offensichtlich neue Ideen bei der kostengünstigen Substitution von Stoffen frei.

- Schließlich ist vorstellbar, daß Unternehmen in Bezug auf Umweltschutz organisationales Lernen initiieren, also die Verbesserung der Lernfähigkeit einer Organisation selbst zum Gegenstand zu machen. Bezogen auf den Umweltschutz ist dies unter betriebswirtschaftlichen Gesichtspunkten dann notwendig, wenn Unternehmen erkennen, daß die Existenz bedroht ist und vor diesem Hintergrund eine grundsätzliche Veränderung des Lernens unabweisbar geworden ist. Als Beispiel kann die Vermittlung von umweltschutzbezogenem Grundlagenwissen in Unternehmen herangezogen werden, die z.B. durch ein hohes Maß an Umweltschädigung, veränderte Konsumentenpräferenzen oder staatliche Sanktionen nachhaltig herausgefordert sind. Je nach Gefährdungslage (oder Chancen) sind unterschiedliche Fähigkeiten oder Kenntnisse zu vermitteln. Personalentwicklung hat in diesem Fall Arbeitsvermögen von Arbeitnehmern zu entwickeln, um umweltschutzbezogene Herausforderungen überhaupt erst zu identifizieren und damit Wege ihrer Bewältigung erschließen zu können. Die strukturelle Aufgabe enthält damit die Vermittlung von Fähigkeiten zur Antizipation und Bewältigung externer umweltschutzbezogener Herausforderungen sowie interner Anforderungen.

\section{Ausblick}

Konzepte des organisationalen Lernens sind nicht im Hinblick auf den Umweltschutz entwickelt worden. Innerhalb der Betriebswirtschaftslehre gelten sie eher als intelligentes Instrument, das eine erhöhte Wettbewerbsfähig- keit durch Anpassungsfähigkeit von Organisationen erzeugen soll. Geht man von einem pragmatischen Wissenschaftsziel der Betriebswirtschaftslehre aus, wonach die bestehenden Problemlagen der Praxis theoretisch erfaßt und ggf. mit Hilfe von Gestaltungsvorschlägen verbessert werden sollen, verspricht die Übertragung der Konzepte des organisationalen Lernens eine Fülle von Möglichkeiten und Instrumenten, den Unternehmenserfolg durch ökonomisch intendierte, strategisch vorbereitete und angepaßte Instrumente der Personalentwicklung bezogen auf den Umweltschutz zu verbessern.

\section{Hans-Gerd Ridder, Hannover}

\section{Literatur}

- Argyris, C./Schön D. A.: Organizational Learning: A Theory of Action Perspective, Reading/Mass, u. a. 1978

- Dodgson, M.: Organizational Learning: A Review of Some Literatures, in: Organization Studies, 14(1993)3, S. 375-394

- Pawlowsky, P.: Betriebliche Qualifikationstrategien und organisationales Lernen, in: Staehle, W. H./Conrad P. (Hrsg.): Managementforschung 2, Berlin / New York 1992, S. 177-237

- Ridder, H.-G.: Grundprobleme einer ethisch-normativen Betriebswirtschaftslehre, in: Pfriem, R. (Hg.): Ökologische Unternehmenspolitik, Frankfurt/M. 1986, S. 52-80

- Ridder, H.-G.: Die Integrationsfähigkeit der Allgemeinen Betriebswirtschaftslehre am Beispiel der Ökonomisierung ökologischer Fragestellungen, in: Freimann, J. (Hg.): Ökologische Herausforderung der Betriebswirtschaftslehre, Wiesbaden 1990, S. 145-159

- Ridder, H.-G.: Unternehmensethik als Instrument der Transformation von Ökologie in Ökonomie. In: Müller-Jentsch W. (Hrsg.): Profitable Ethik - effiziente Kultur, München/Mering 1993 S. $107-130$

\section{Aus dem VöW- Studierendenkreis}

\section{Wangerooge und die Folgen}

Seit 1991 setzt sich der Studierendenkreis der VÖW für eine ökologische Veränderung der wirtschafts- und sozialwissenschaftlichen Lehre ein. In der Wangerooger Erklärung (INFORMATIONSDIENST 2/94) haben Mitglieder des Studierendenkreises Kriterien für eine verbindliche Festlegung von Mindestanforderungen an eine ökologische wirtschaftswissenschaftliche Ausbildung aus ihrer Sicht formuliert. In der Wangerooger Erklärung werden Forderungen an die Didaktik, die Volkswirtschaftlehre und die Betriebswirtschaftlehre unterschieden. Die ausführlichere Dokumentation zum Workshop, die u. a. Texte zu den Einstiegsvorträgen, Erläuterungen und Begründungen zur Wangerooger $\mathrm{Er}$ klärung enthält, ist für DM 20,- plus Versandkosten in der Geschäftsstelle erhältlich.

Die Wangerooger Erklärung wird bislang von Institutionen und Verbänden wie z: B. B. A.U.M. e.V., Hamburg, UnternehmensGrün, Global Challenge Network e. V. sowie von über 30 ProfessorInnen und wissenschaftlichen Mitarbeitern an Hochschulen und Fachhochschulen unterstützt.

Im Mai und Juni beteiligten sich Universitäten und Hochschulen in Hamburg, Göttingen, Mainz, Mannheim, Oldenburg und Berlin an den Aktionstagen zur Wangerooger Erklärung, u. a. an der Uni Göttingen: Vorlesungsreihe über ausgewählte betriebs- und volkswirtschaftliche Themen zu Umwelt und Wirtschaft mit abschließender Podiumsdiskussion, Uni Hamburg: Podiumsdiskussion zur Ökologisierung der Wirtschaftswissenschaften, Unterschriften und Plakataktionen an der Uni Mainz. In Berlin nahmen Anfang Juni Studierende der dortigen Hochschulen an den Berliner Umwelttagen der Fachhochschule für Wirtschaft teil. In diesem Rahmen wurde eine zentrale Pressekonferenz zu den Aktionen und Reaktionen auf die Wangerooger Erklärung durchgeführt sowie eine Podiumsdiskussion mit Beteiligung von Lehrenden der Berliner Universitäten und Fachhochschulen zur Wangerooger Erklärung und zu Möglichkeiten der praktischen Umsetzung an Universitäten und Hochschulen.
Auch im nächsten Semester werden weitere Aktionstage an den Hochschulen durchgeführit. Auf der nächsten Herbstakademie des Studierendenkreises ist dann eine inhaltliche Überarbeitung der Wangerooger Erklärung unter Einbezug der Reaktionen und Anregungen der Lehrenden und der Studierenden (durchgeführte Unterschriftenaktionen, Ergebnisse der Hochschulaktionstage) vorgesehen.

Die nächste Herbstakademie des Studierendenkreises wird vom 23.-26.11. in St. Gallen stattfinden. Das Schwerpunktthema ist Handlungsspielräume und Handlungsgrenzen ökologischer Unternehmenspolitik. Bei Interesse an der Vorbereitung der Herbstakademie bitte in der $\mathrm{Ge}$ schäftsstelle melden (1. Vorbereitungstreffen vor Ende August)

Voraussichtlich im Januar/Februar 1995 wird der Studierendenkreis der VÖW eine WerkstattTagung durchführen, bei der Studierende und Lehrende gemeinsam konkrete Umsetzungsstrategien zur Implementierung ökologischer Inhalte in die Lehre entwickeln. Informationen sind in der Geschäftsstelle erhältlich.

Kirsten Mintert, Berlin 
(c) 20I0 Authors; licensee IÖW and oekom verlag. This is an article distributed under the terms of the Creative Commons Attribution Non-Commercial No Derivates License (http://creativecommons.org/licenses/by-nc-nd/3.o/), which permits unrestricted use, distribution, and reproduction in any medium, provided the original work is properly cited. 